\title{
A Hierarchical Assessment Method using Bayesian Network for Material Risk Detection on Green Supply Chain
}

\author{
Benjamin P.-C. Yen, Bingcong Zeng \\ School of Business, The University of Hong Kong, Hong Kong, China \\ ( benyen@business.hku.hk, bczeng@hku.hk )
}

\begin{abstract}
Today's social awareness of environmental protection presents the electronic companies with an irreversible trend towards green manufacturing. It raises harsh requirement for the sourcing process and imposes unprecedented pressure to the QA system, majorly due to the risk of hazardous material. As QA procedures are becoming more complicated for coping with increasing material risk and meanwhile the time and resource available are tightly constrained, the development of an effective mechanism for material testing turns up to be a critical issue. In this study, a hierarchical material risk assessment approach is proposed based on FMEA framework. Taking into account the risk occurrence, the difficulty in detection and the severity the risk causes, it enables companies to estimate their material risks dynamically using Bayesian network. With its help, companies can assess and prioritize the material risk in a systematic and efficient manner which will drive QA towards a more high-performance process.
\end{abstract}

Keywords - material risk, green supply chain, decision method

\section{INTRODUCTION}

The depletion of non-renewable resources, global warming, the pollution of air, soil and water beyond the Nature's carrying limits, such terms condemning the harm of modern industry on the natural environment are overwhelming in the headlines of mass media. Especially for the electronics industry, the companies are subjected to tremendous pressure in the light of the push for green products. On one hand, they are competing in a global market in which customers are being more meticulous and they demand high-quality but low-cost products. On the other hand, under pubic pressure, the companies have to be good environmentalists in order to gain trust from the customers. Undoubtedly, the notion of environmental protection has been widely disseminated in the society, and consumers have consistently expressed the willingness that they would like to pay a premium for the "green" products. Most of surveys conducted showed that consumers are willing to pay $10 \%$ extra if the products they are purchasing are environmental friendly [1]. It is right reflecting consumers' increasing concerns on the impact of the products upon the environment.

As a response, market-based environmental initiatives were emerged in 1990s, which are chiefly concentrated on the consumers side and emphasize green marketing as a feasible way to achieve sustainability in the context of supply chains [2]. However, in terms of striving for sustainable development, merely focusing on the downstream part is insufficient. A comprehensive perspective of a company's environmental impact needs attention on its supply side also [3]. Green supply offers a significant complement to those initiatives that unconsciously confine themselves in satisfying the green consumers only [4].

The concept of green supply is mainly referred to supply management activities with attempt to improve the environmental performance of purchased inputs as well as of the suppliers and their operational processes [5]. In fact, the environmental impact of the supply side is not just limited in raw material and component procurement, but also includes suppliers' manufacturing processes and logistics transportation. Aware of this, Bowen et al. in their study differentiated green supply into two major categories: "product based green supply" and "supplier management" [5]. Product based green supply aims to through cooperation with the suppliers get rid of the hazardous substances in the raw materials, thus ensuring the final products truly green. Supplier management goes forward one step ahead and it tries to influence and even to intervene in the supplier's actives with intention to achieve the expected environmentally sound performance in the relationship with the supplier.

Given the strategic importance and valuable significance of green supply, there continually emerge a number of directives released by institutions and governments, regarding the products, raw materials and components to be purchased, in which the use of certain hazardous materials are limited or banned [6]. One of the most influential and famous directives is RoHS. The European Union Reduction of Hazardous Substance (EU RoHS) is a set of environmentally based regulations legislated by the EU and has taken effect on 1 July 2006, which is required to be enforced and becomes law in each member state of EU. RoHS compliance is applicable for products to be sold in the EU region. This directive restricts the use of six hazardous materials in the manufacture of various types of electronic and electrical equipments. The restricted substances encompass lead $(\mathrm{Pb})$, Mercury $(\mathrm{Hg})$, Cadmium (Cd), hexavalent chromium $(\mathrm{Cr} 6+)$, polybrominated biphenyls (PBB) and polybrominated diphenyl ether (PBDE). Among the six, lead removal will cause the greatest impact on PCB (printed circuit board) assembling process where lead is used in the attachment of electronic components through leaded solder. Consequently, it can hugely affect nearly all the electronic products. 
When it is adopted, the pressure of RoHS has been hanging over every sector of the electronic industry. The quality assurance (QA) exemplifies the serious difficulties, with more works to be done in IQC (Incoming Quality Control) and more complicated material testing procedures needed in order to prevent hazardous chemicals from being interfused in the final products. Despite the fact that suppliers are obligated to provide material declaration, it is still far from enough to be convinced that the raw materials are all complied with the RoHS directive. Potential material risk can surge if the components contain the parts with high suspect in violation and the suppliers involved are from multiple levels. In cases of concern, detailed sampling and testing are definitely required. Therefore the workload for QA has been tremendously increased. Eager to help QA operate more efficiently, we would like to propose a hierarchical material risk assessment (HMRA) approach in this paper. The aim of HMRA is to enable companies to estimate and prioritize the material risks in a systematic and efficient way so that the performance of QA process can be greatly improved. A demo case of an electronic manufacturer applying HMRA in material risk assessment is elaborated in this study and as an application example we hope it can comprehensively show the feasibility and validity of this approach.

\section{THEORETICAL BASIS AND METHODOLOGY}

The implementation of environmental directives has incurred numerous new risks of raw materials. Taking RoHS as an example, the directive limits and bans the use of six hazardous substances in the electronic products, thus resulting in a much higher standard for QA process in which at least six new kinds of risks are imposing threat to raw materials. More complicated component testing procedures are required to mitigate the increasing material risks. Under the backdrop of this, a hierarchical material risk assessment (HMRA) approach is proposed here. It is a mechanism design with attempt to confer a systematic approach to assess and prioritize material risks in the QA process. The material risks we are concerned about in this study are referred to the potential quality problems of raw materials provided by suppliers who fail to commit the quality declaration. Depended on different products with different complexity, the profile of material risks can vary in a wide range with respect to the scale, possibly from a relatively small set of risks with only 5 to 10 checkpoints up to thousands of risk nodes with huge hierarchies. Moreover, the material risks are not isolated, and they can be correlated following the relationships in BOM (Bill of Material) table. The risk level of a part may affect the risk of quality of the product. Given multiple material risks and the complicated relationships among them, how to assess the risk levels scientifically by taking into account the correlation factor of the material risks and then assign the correct priority to them has become a key issue on the current executive agenda. To address this issue, a systematic procedure for risk assessment is needed, and HMRA provides a feasible avenue.

\section{A. Theoretical Basis: FMEA Framework}

The development of HMRA is on the basis of FMEA (the failure models and effect analysis) framework. FMEA is a systematic and proactive engineering quality method for analysis of potential failure modes within a system. Although it has been widely used in manufacturing industries in various phases of the product life cycle, it is shown that FMEA is most effective when it is applied in the early stage of the product development [7]. FMEA contains two key concepts. The first one is failure mode which is referred to a manner that any errors or defects occur or will occur in a component, subsystem or process, especially those that affect the customers. It can be potential or actual. The failure mode of interest is subjected to the specific component, subsystem, process and product under consideration, and also determined by the failure history of the similar cases documented in the past. Another key concept is effects analysis. It refers to a series of investigations on the consequences of those failures. Actually, different failure modes can impact each other, and in this case it will increase the difficulty of the effects analysis.

Fig.1 below illustrates a FMEA cycle which is typically applied in risk assessment. Generally speaking, FMEA assesses the potential failure or risk from 3 aspects, including severity, occurrence, and detection. Severity is a rating corresponding to the seriousness of an effect of a potential failure mode, and it measures the influential extent to which the consequence can be caused by the risk. Occurrence refers to the probability of the failure, and it describes how likely the risk would happen. Detection is associated with the difficulty to detect the failure, and it depicts how hard the process is in ensuring the happening of the failure. The risk priority number (RPN) integrates the aspects of severity, occurrence and severity by the multiplication of them. Since FMEA is well fitted to solve the material risk assessment problem, we apply it here in the context of quality assurance and based on it develop the HMRA approach.

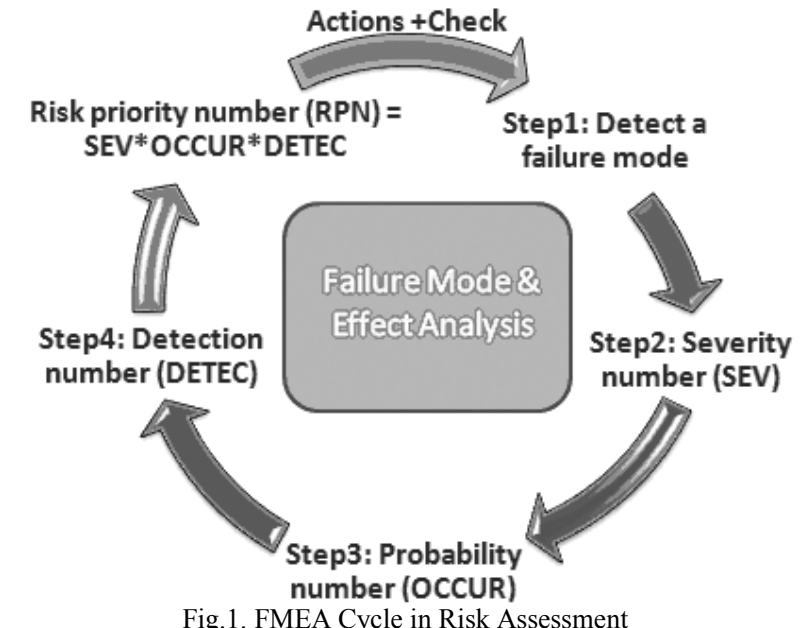

Fig.1. FMEA Cycle in Risk Assessment 


\section{B. HMRA Approach}

To certain extent, HMRA can be viewed as an extension of FMEA applied in material risk assessment in the QA process. It inherits the systematic framework of FMEA in failure mode analysis and extends the analysis by taking into consideration the correlation effect among the material risks.

The HMRA approach comprises a number of interrelated steps which are summarized in Fig. 2. The order presented is suggested by us and hence it may not necessarily reflect the sequence in practice. In real situation, for instance, the impact evaluation can proceed after the evaluation of the detection, or maybe they can be conducted simultaneously also. Regarding the workflow planning, HMRA gives flexibility to QA planners so that the planners are allowed to arrange the implementation steps according to the specific cases.

In HMRA, the key steps involve determining the score of severity, occurrence and detection. Under the pressure of RoHS, if the product is tested out to be interfused with restricted hazardous chemicals, it will result in huge amount of penalty. The financial loss is the major negative impact of the material risks. If there exists some agreement between the manufacturer and supplier that the supplier would shoulder the penalty in case that the hazardous substance is found in the parts it supplies, then the severity of the risk for the manufacturer will be greatly reduced. Such agreement is normally stated in the quality declaration. However, the supplier may not agree to suffer the penalty by itself and thereby in most cases the supplier and manufacturer are required to share the financial loss if the material risk happens. But if the supplier does not provide quality declaration for RoHS, the severity of material risk for the manufacturer will be very high, and it implies that the manufacturer may need to pay the huge amount of the penalty for the supplier who violates the RoHS directive. Based on the discussion above, we have made a reference score table for the material risk severity which is shown in TABLE I.

TABLE I

\begin{tabular}{llc}
\multicolumn{4}{c}{ A Reference Score Table for the Material Risk SEV } & \multicolumn{1}{c}{ Indicator } & \multicolumn{1}{c}{ Score } \\
\hline Risk Level & \multicolumn{1}{c}{$\begin{array}{l}\text { The supplier does not submit the quality } \\
\text { declaration for RoHS }\end{array}$} & 100 \\
\hline High & $\begin{array}{l}\text { The supplier has submitted the quality } \\
\text { declaration for RoHS, however, the supplier } \\
\text { and manufacturer need to share the cost if the } \\
\text { material risk happens }\end{array}$ & 10 \\
The supplier has submitted the promise for & \\
Low & $\begin{array}{l}\text { RoHS, in which the supplier agree to shoulder } \\
\text { most of the penalty if the material risk happens } \\
\text { due to the parts it supplies. }\end{array}$ & 1 \\
\hline
\end{tabular}

TABLE I aims to illustrate an example, from the perspective of an electronic manufacturer, to measure the severity of material risk under the effect of the RoHS directive. According to it, the risk level is divided into three levels, and the corresponding score will be assigned under different situation. The sore of SEV can be 100 or
10 or 1 . The score scale can be adapted to different cases, and it would be much better if it is suggested by risk experts according to the specific situation. The scale of 100,10 and 1 here is a demonstrated example, which reflects an exponential relationship in quantity existing between the severity of the negative consequence brought by the risk and the corresponding risk level. Taking a reference of it, companies can make their own severity score tables depended on the specific cases they are facing in the practice.

Assessing the OCCUR of material risk is another core section in the HMRA approach. As a matter of fact, the material risks are correlated in terms of OCCUR. The risk in parts may cause the problem in the product. Fig.3 demonstrates a hierarchical relationship among the major pieces of equipments in an air conditioner. It can be inferred that if the likelihood in condenser to violate RoHS becomes high, the likelihood in chiller will probably increase because of the correlation effect, and this effect may expand to the subsystem level and even the system level through the relationship path.

Given that, the material risks can be presented in a causality network by linking them according to their relationships in BOM. BOM is hierarchical in nature with the top level representing the finished goods and the subsequent levels including components, units and parts. Consequently, the causality network is organized in a hierarchical structure, covering across multiple levels of suppliers in the supply chain. Fig.4. shows an example of a causality network of material risks.

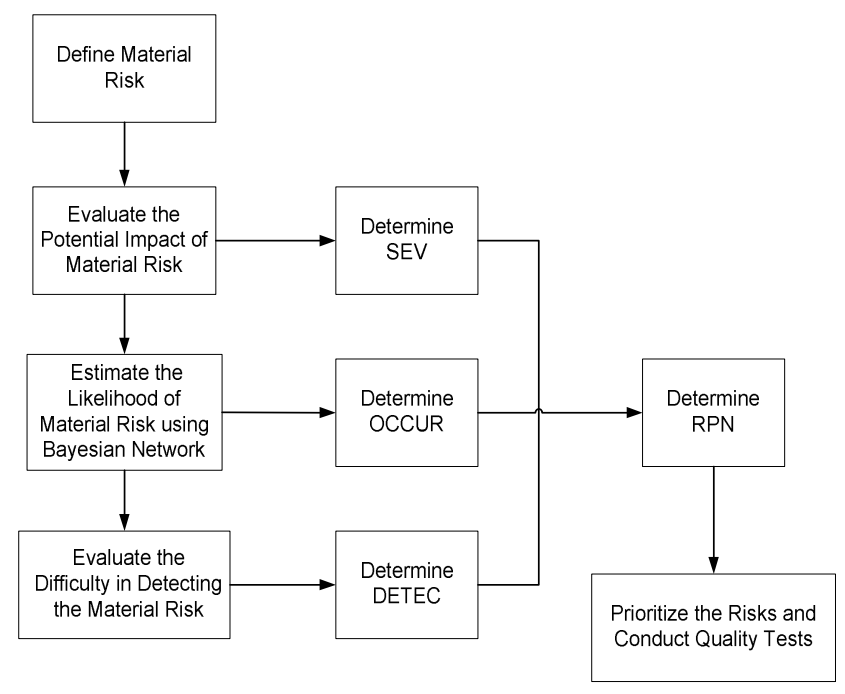

Fig.2. Suggested Workflow of HRMA Approach

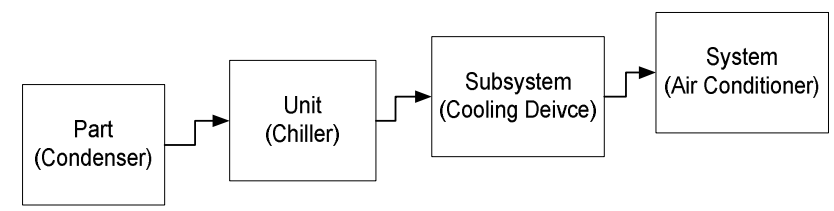

Fig.3. Hierarchical Structure of components in an air conditioner 


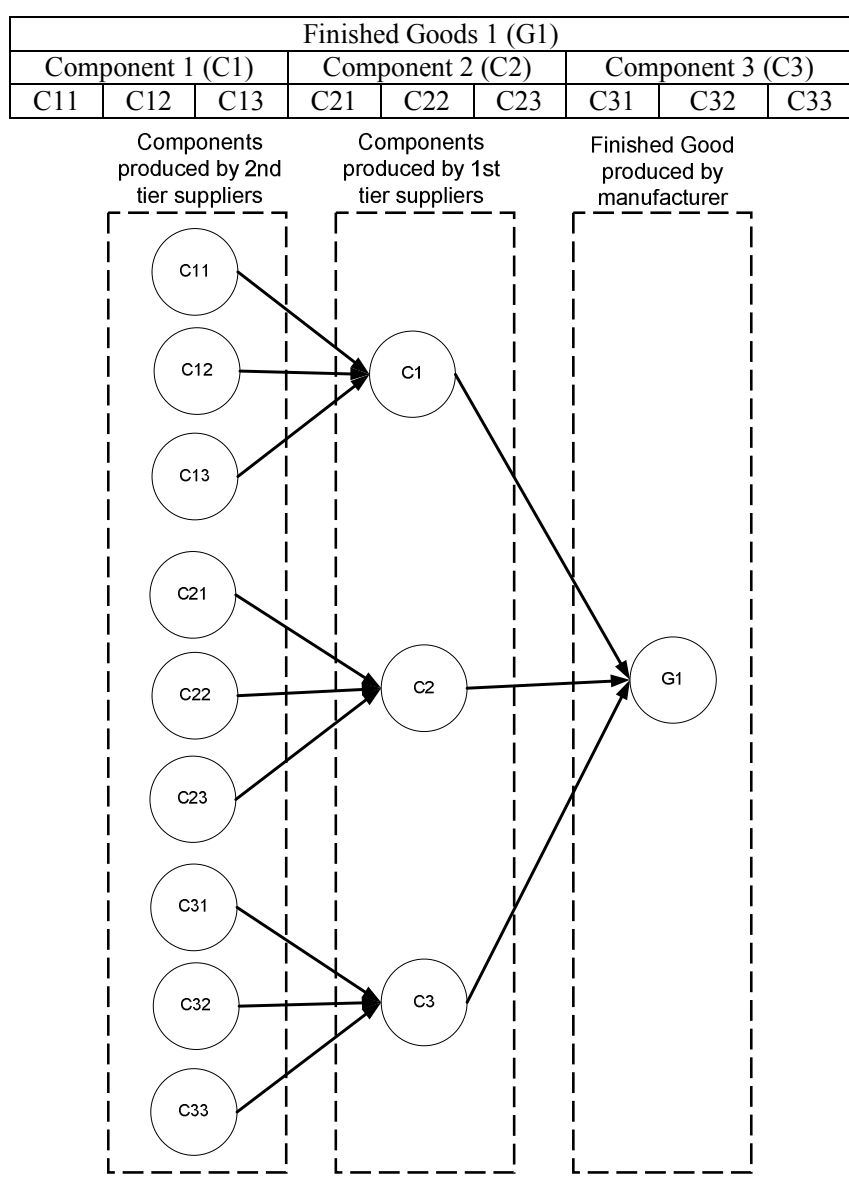

Fig.4. Causality Network of Material Risks

In the network shown in Fig.4, each node stands for a material risk that may cause RoHS violation. The OCCUR of risk is classified into high $(\mathrm{H})$, mediate $(\mathrm{M})$ or low (L), which follows a discrete probability distribution with 3 values. So the summation of the probability must be equal to 1 :

$$
p_{H}+p_{M}+p_{L}=1
$$

TABLE II suggests a reference score table which provides a guidance in determining the OCCUR for material risks.

TABLE II

A Reference Score Table for the Material Risk OCCUR

\begin{tabular}{|c|c|c|}
\hline Risk Level & Indicator & Score \\
\hline High & $\begin{array}{l}\text { In material sampling test, the material } \\
\text { contains one of hazardous substances which } \\
\text { level is high than } 80 \% \text { of the warning value } \\
\text { stated in the rules of RoHS. }\end{array}$ & 100 \\
\hline Moderate & $\begin{array}{l}\text { In material sampling test, the material } \\
\text { contains one of hazardous substances which } \\
\text { level is between } 20-80 \% \text { of the warning } \\
\text { value stated in the RoHS directive. }\end{array}$ & 10 \\
\hline Low & $\begin{array}{l}\text { In material sampling test, the material } \\
\text { contains one of hazardous substances which } \\
\text { level is below } 20 \% \text { of the warning value } \\
\text { stated in the rules of RoHS. }\end{array}$ & 1 \\
\hline
\end{tabular}

To implement the approach in this phase, suppliers are required to submit a risk report. Based on the risk report submitted by the suppliers, the manufacturer is able to estimate occurrence of material risks through Bayesian update. Supposing the $1^{\text {st }}$ tier suppliers report that $C_{1}=M, C_{2}=L, C_{3}=M$, then the score of occurrence for $\mathrm{G} 1$ will be

$$
\begin{aligned}
& \text { OCCUR }=100 \times P_{H \mid C_{1}=M, C_{2}=L, C_{3}=M}+10 \times P_{M \mid C_{1}=M, C_{2}=L, C_{3}=M} \\
& +1 \times P_{L \mid C_{1}=M, C_{2}=L, C_{3}=M}
\end{aligned}
$$

In the QA process, there exists difference regarding the difficulty in testing different raw materials. In some material tests, in order to accurately test out the hazardous ingredient, the component needs to be separated and even damaged; whereas in other tests, the task can be done through simply being scanned by an intelligent device. TABLE III provides a reference in judging the DETEC for the material risk.

TABLE III

A Reference Score Table for the Material Risk DETEC

\begin{tabular}{lll}
\multicolumn{4}{c}{ Indicator } & Score \\
\hline Risk Level & $\begin{array}{l}\text { The accurate test needs damage of the } \\
\text { component } \\
\text { High }\end{array}$ & $\begin{array}{l}\text { The accurate test needs tools or requires } \\
\text { skills or takes long time. }\end{array}$ \\
The accurate test can be conducted directly & 10 \\
Low & \begin{tabular}{l} 
and easily \\
\hline
\end{tabular}
\end{tabular}

After determining the scores for material risk in SEV, OCCUR and DETEC, the RPN can be calculated by multiplying the three scores:

$$
\mathrm{RPN}=\mathrm{SEV} \times \text { OCCUR } \times \text { DETEC }
$$

During IQC, the components with high RPN should be assigned high priority in the material test. When under the circumstance that the test budget and time are in shortage, the QA managers can consider delay the test of the components with relatively low RPN, and even temperately take them out of the testing schedule in order to secure the normal operation in the QA process.

\section{AN APPLICATION EXAMPLE}

In this section, we would like to use an application example to demonstrate the feasibility and validity of HMRA in material risk assessment. The business scenario is set in an electronic company which is manufacturing personal computers (PCs) as its major business. The company has a number of suppliers, and generally its suppliers can be differentiated into two tiers based on the components they supply in the BOM table. To make it clear, we simplify the situation by assuming that the PC manufacturer is only interested in assessing the material risks of three components, including $\mathrm{C} 1$ : mother board; C2: display card; and C3: CPU. Their BOM structures are shown as follows:

$\begin{array}{ccc}\text { C1: Mother board } & \text { C2: Display card } & \text { C3: CPU } \\ \text { C11: PC board } & \text { C21: Display memory } & \text { C31: Chip set } \\ \text { C12: Connector } & \text { C22: Display chip } & \text { C32: Cache } \\ \text { C13: Heat fan } & \text { C22: Fan } & \text { C33: Shell }\end{array}$


In this example, the causality network of material risks is the same as the one shown in Fig. 4 as regard the topological structure. The three components in the $1^{\text {st }}$ tier are supplied by three different suppliers. The suppliers of display card and CPU both have the quality declaration for RoHS and they agree to shoulder the penalty if the restricted hazardous substance is found in the parts they supply. Nonetheless, the supplier of mother board due to some reason cannot provide the quality declaration for the RoHS directive. Given the information above plus the criteria stated in TABLE $\mathrm{I}$, it can be judged that $\operatorname{SEV}(\mathrm{C} 1)=100, \operatorname{SEV}(\mathrm{C} 2)=1$, and $\operatorname{SEV}(\mathrm{C} 3)=1$.

Based on the risk report provided by suppliers, it is said that the occurrence level of PC board C11 is high $(\mathrm{H})$, connector C12 is media (M), and heat fan C13 is low (L). The conditional probability of the material risk of mother board $\mathrm{C} 1$ given $\mathrm{C} 11=\mathrm{H}, \mathrm{C} 12=\mathrm{M}$ and $\mathrm{C} 13$ can be calculated through the historical data stored in the enterprise risk database. Suppose that the below information is available:

\begin{tabular}{|l|l|l|l|l|}
\hline C11 & C12 & C13 & C1 & Frequency \\
\hline H & M & L & H & 1031 \\
\hline H & M & L & M & 8967 \\
\hline H & M & L & L & 3662 \\
\hline & & & Sum: & 13660 \\
\hline
\end{tabular}

Thus,

$$
\begin{aligned}
& \operatorname{OCCUR}(\mathrm{C} 1)=100 \times P_{H \mid C_{1}=H, C_{2}=M, C_{3}=L}+10 \times P_{H \mid C_{1}=H, C_{2}=M, C_{3}=L} \\
& +1 \times P_{H \mid C_{1}=H, C_{2}=M, C_{3}=L}=100 \times \frac{1031}{13660}+10 \times \frac{8967}{13660}+1 \times \frac{3662}{13660} \\
& =14.38
\end{aligned}
$$

Following the similar approach, we can dynamically update the occurrence of $\mathrm{C} 2$ and $\mathrm{C} 3$ by using the information in suppliers' risk report and the historical data in the risk database. To reduce redundancy of description, we would like to give out the result without showing the computation process. After a series of computation by somehow, it is worked out that $\operatorname{OCCUR}(\mathrm{C} 2)=12.56$, and $\operatorname{OCCUR}(\mathrm{C} 3)=10.77$. The occurrence scores of the three components imply that motherboard is most risky component in terms of the likelihood of material risk, but their difference is within a small range.

Actually, the material tests for the three components all require professional skills and considerable time. But among them, the examination for CPU is most difficult since the test needs to crack the shell of the component for sampling, and as a result the tested CPU would be damaged. Through applying the criteria listed in TABLE III, it can be judged that $\operatorname{DETEC}(\mathrm{C} 1)=10$, $\operatorname{DECTEC}(\mathrm{C} 2)=10$, and DECTEC $(\mathrm{C} 3)=100$.

Therefore, by Formula (2), we can obtain the risk priority numbers for the three components respectively:

$\mathrm{RPN}(\mathrm{C} 1)=14380, \mathrm{RPN}(\mathrm{C} 2)=125.6, \mathrm{RPN}(\mathrm{C} 3)=1077$

In the model of HMRA, RPNs, as the final measurement, assess the material risks through integrating the aspects of severity, occurrence and severity. The result of the example shows that the mother board $(\mathrm{C} 1)$ has the highest risk to violate the RoHS directive among the three components under consideration. Hence, it implies that special attention should be put on the mother board tests during the IQC process. If possible, the manufacturer may consider increasing the number of tests for mother boards, and the related tests should be assigned to high priority so that they can be arranged at the top of the testing schedule. Moreover, regarding risk mitigation, the manufacturer can negotiate with the supplier and try to persuade it to offer quality declaration. If the supplier still fails to provide quality declaration, the manufacturer may consider switching to another more reliable motherboard supplier.

\section{CONCLUSION}

Increasing social awareness of environmental protection makes governments continuously legislate mandatory compliances or directives with a variety of restrictions on the use of certain hazardous substances. By setting high violation penalty, the directives have imposed tremendous pressure on the electronic companies, especially on their QA process. The HMRA approach is proposed in this paper with aim to provide an effective mechanism for material testing to cope with those environmental directives. HMRA can be thus viewed as a systematic procedure to assess and prioritize the material risks in the QA process. It is developed upon FMEA, a well-accepted framework in manufacturing industry, therefore HMRA is easy to implement. Furthermore, it innovatively takes into account the correlation effect of the material risks in risk assessment, and uses Bayesian inference to estimate the risk occurrence. HMRA is shown to be feasible and effective in this study. We hope the HMRA approach can give inspiration to the practitioners and benefit the continuous improvement for the QA process.

\section{REFERENCES}

[1] S. G. Shina, Green electronics design and manufacturing: implementing lead-free and RoHS-compliant global products. New York: McGraw-Hill, 2008.

[2] K. Peattie, "Towards Sustainability: The Third Age of Green Marketing," Marketing Review, vol. 2, pp. 129-146, 2001.

[3] R. Welford, Corporate environmental management 3: towards sustainable development London: Earthscan, 2000.

[4] L. Preuss, The green multiplier: a study of environmental protection and the supply chain. New York: Palgrave Macmillan, 2005.

[5] F. E. Bowen, P. D. Cousins, R. C. Lamming, and A. C. Faruk, "The role of supply management capabilities in green supply," Production and Operations Management, vol. 10, pp. 174-189, 2001.

[6] J. D. Linton, R. Klassen, and V. Jayaraman, "Sustainable supply chains: An introduction," Journal of Operations Management, vol. 25, pp. 1075-1082, 2007.

[7] R. E. McDermott, R. J. Mikulak, and M. R. Beauregard., The basics of FMEA. New York: CRC Press, 2009. 